\title{
VIBRATIONS OF NON-UNIFORM SPHERICAL SYSTEMS
}

\author{
G. SEVERNE \\ Fakulteit Wetenschappen, Vrije Universiteit Brussel, Brussels, Belgium \\ and
}

A. KUSZELL

Institute of Nuclear Research, Warsaw-Swirck, Poland

\begin{abstract}
Dispersion relations have been obtained and analyzed for a non-uniform, non-rotating spherical gravitational system. A restriction to short wavelengths makes it possible to consider a linearized form of the collisionless Boltzmann equation, differing from that for homogeneous systems by the appearance of a term expressing the effect of the mean self-gravitational field upon the motion. The mean field affects the radially directed wave perturbations, with a breaking of symmetry.

Inwardly and outwardly directed modes have quite different propagation characteristics, inward modes being preferentially propagated. Locally, the stability of the system is enhanced due to the effect of the mean field.
\end{abstract}

From the point of view of statistical mechanics, and more particularly with reference to the crucial problems of the approach towards a quasi equilibrium and the degree of applicability of a thermodynamic description, it must be recognized that there is still much to accomplish in the study of gravitational systems. A major difficulty is the existence of a strong field and of a large inhomogeneity. The motivation for the work now being reported (Severne and Kuszell, 1975, further denoted as Ref. I) was the isolation of a strong field effect, which is simple enough to be treated with reasonable rigour.

To this end, we have reconsidered the classical problem of the vibrations of a stellar system, as treated first by Lynden-Bell (1962). We however take a non-rotating spherical system, so that in the linearized collisionless Boltzmann equation

$$
\frac{\partial f}{\partial t}+\mathbf{v} \cdot \frac{\partial f}{\partial \mathbf{r}}+\mathbf{F} \cdot \frac{\partial f^{(0)}}{\partial \mathbf{v}}(\mathbf{r}, \mathbf{v})+\mathbf{F}^{(0)}(\mathbf{r}) \cdot \frac{\partial f}{\partial \mathbf{v}}=0
$$

there now appears a mean field term, in $\mathbf{F}^{(0)}(\mathbf{r})$. Here $f(\mathbf{r}, \mathbf{v}, t)$ is the perturbed mass distribution in phase space. The acceleration field $\mathbf{F}(\mathbf{r}, t)$ is self-consistently determined by the Poisson equation

$$
\operatorname{div} \mathbf{F}(\mathbf{r}, t)=-4 \pi G \int \mathrm{d} \mathbf{v} f(\mathbf{r}, \mathbf{v}, t) .
$$

The perturbations are with respect to the stationary but inhomogeneous, i.e. position dependent state: $f^{0}(\mathbf{r}, \mathbf{v}), F^{0}(\mathbf{r})$.

The analysis remains quite simple if we restrict attention to plane waves, of wavelength small on the scale of the inhomogeneity

$$
\lambda \ll L_{H}
$$


The scale $L_{H}$ itself is determined by the unperturbed state $f^{(0)}, F^{(0)}$, and is thus of the order of the dimensions of the system. The restriction (3) to perturbations of small wavelength means that $f^{(0)}$ and $F^{(0)}$ can locally be taken as constant. One can then, in the usual manner, take advantage of a Fourier-Laplace transformation, according to

$$
\phi_{\mathbf{k}}(z)=\int_{0}^{\infty} \mathrm{d} t \int \mathrm{d} \mathbf{r} e^{-i(\mathbf{k} \cdot \mathbf{r}-z t)} \phi(\mathbf{r}, t) .
$$

When one further integrates over the velocity components perpendicular to $\mathbf{k}$

$$
\begin{aligned}
& \bar{f}_{\mathbf{k}}(u, z)=\int \mathrm{d} \mathbf{v}_{\perp} f_{\mathbf{k}}(\mathbf{v}, z), \\
& u=\mathbf{k} \cdot \mathbf{v} / k, \quad \mathbf{k} \cdot \mathbf{v}_{\perp}=0,
\end{aligned}
$$

the collisionless Boltzmann equation becomes

$$
i(k u-z) f_{\mathbf{k}}(u, z)-h_{\mathbf{k}}(u)+F_{\mathbf{k}}(z) \frac{\partial f^{(0)}}{\partial u}=-\frac{\mathbf{F}^{(0)} \cdot \mathbf{k}}{k} \frac{\partial \vec{f}_{k}(u, z)}{\partial u},
$$

with $F_{\mathbf{k}}(z)$ the Fourier-Laplace transform of the field, according to (4), and $h_{\mathbf{k}}(u)$ the perturbation at initial time:

$$
h_{\mathbf{k}}(u)=\bar{f}_{\mathbf{k}}(u, t=0) \text {. }
$$

Equation (6) differs from that for homogeneous systems by the appearance of a r.h.s. Thus, due to the mean field, $\bar{f}_{\mathbf{k}}$ is determined by a differential equation instead of by an algebraic equation. The differential equation is however elementary, its solution being

$$
\begin{aligned}
& f_{\mathbf{k}}(u, z)=e^{-i \alpha(u)} \int_{-\infty \mathbf{S g n}_{\mathbf{F}}^{(0) \cdot \mathbf{k}}}^{u} \mathrm{~d} u^{\prime} e^{i \alpha\left(u^{\prime}\right)} g\left(u^{\prime}, z\right), \\
& \alpha(u)=\frac{k}{\mathbf{F}^{(0)} \cdot \mathbf{k}}\left(\frac{1}{2} k u^{2}-u z\right), \\
& g(u, z)=\left[h_{\mathbf{k}}-\mathbf{F}_{\mathbf{k}}(z) \frac{\partial f^{(0)}}{\partial u}\right] \frac{k}{\mathbf{F}^{(0)} \cdot \mathbf{k}} .
\end{aligned}
$$

Due to the $\exp (i \alpha)$ factor of the integrand, one will expect to encounter complex error or related functions, whatever the velocity distribution.

Substitution of (8) into the transform of the Poisson Equation (2) yields for the perturbation to the mean field an expression of the form

$$
F_{\mathbf{k}}(z)=\frac{1}{D(\mathbf{k}, z)} H_{\mathbf{k}}(z)
$$

and the vibration modes are thus determined by the solutions of a dispersion relation

$$
D(\mathbf{k}, z)=0 \text {. }
$$


$D$ plays the role of a dielectric function for our problem. $H_{\mathbf{k}}(z)$ expresses transient effects from the initial perturbation (7).

To discuss the vibration modes, it is indispensable to specify the velocity distribution. For the Maxwellian,

$$
f^{(0)}(\mathbf{r}, \mathbf{v})=\varrho_{0}(\mathbf{r})[\sqrt{2 \pi} \sigma(\mathbf{r})]^{-3} \exp \left[-v^{2} / 2 \sigma(\mathbf{r})\right],
$$

and in terms of the parameters

$$
\begin{aligned}
& \kappa=k / k_{0}, \quad \varepsilon=\left|\mathbf{F}^{(0)} \cdot \mathbf{k}\right| / k \sigma \omega_{0}, \\
& \omega_{0}=(4 \pi G \varrho)^{1 / 2}, \quad s=\operatorname{Sgn}\left(\mathbf{F}^{(0)} \cdot \mathbf{k}\right),
\end{aligned}
$$

the dispersion relation takes the explicit form:

$$
\begin{aligned}
& D=1+\frac{\mathrm{d} Z / \mathrm{d} \zeta}{2\left(\kappa^{2}+i s \kappa \varepsilon\right)}=0 \\
& Z=2 i \exp \left(-\zeta^{2}\right) \int_{-\infty}^{i \zeta} \mathrm{d} t \exp \left(-t^{2}\right), \quad \zeta=\frac{z / \omega_{0}}{\sqrt{2 \kappa^{2}+2 i s \kappa \varepsilon}} .
\end{aligned}
$$

In $Z$ one will recognize the plasma dispersion function. The standard results for a homogeneous unperturbed state and the Maxwellian distribution are recovered simply by annulling the field parameter $\varepsilon$.

The dispersion relation (16) allows for an infinity of vibration modes. A detailed discussion is to be found in Ref. I. Here we present only the main results.

Having separated the complex frequency $z$ into real and imaginary parts

$$
z=\omega-i \eta
$$

it is essential to distinguish inward waves, whose radial projection is directed along the field and for which $s \omega>0$, from outward waves, directed against the field and such that $s \omega<0$. As could $a$ priori have been expected, a first effect due to the field is a breaking of symmetry: inward and outward waves have quite different propagation characteristics, while azimuthal waves, for which $\varepsilon=0$, are unaffected by the field. Figures 1 and 2 give the computed curves for the reduced frequency $\omega / \omega_{0}$ and damping $\eta / \omega_{0}$ for different modes (roman label) and values of $\varepsilon$ (latin label). For inward modes (+ index), the frequency increases with the field while the damping decreases (except for the central ' 0 ' mode). For outward modes the converse holds: there is thus preferential propagation of inwardly directed perturbations.

However, as in the field free calculation, the damping remains quite large. It is in fact questionable if these waves can be of physical significance. The relevant parameter is the relative damping $\eta / \omega$, which on Figure 3 is given only for the preferentially propagated inward modes. While the field does have a large effect, for realistic conditions the field parameter is limited to $\varepsilon \lesssim 3$. Moreover the scaling condition(3) imposes a lower limit on $\kappa$, and one is led to conclude that the relative damping remains in excess of 0.3 (e-folding time of 0.5 period). This is far from small. Other velocity 


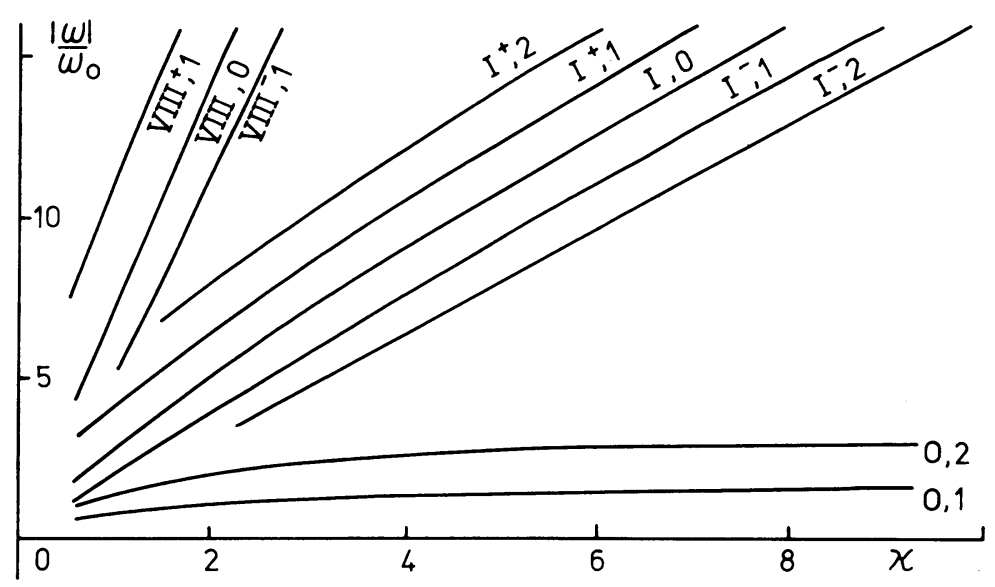

Fig. 1. Dispersion curves for the maxwellian velocity distribution. The curves are labelled by their mode index (roman numeral), and by the value of the field parameter $\varepsilon$ (latin numeral). The superscripts \pm indicate inward $(+)$ or outward $(-)$ modes.

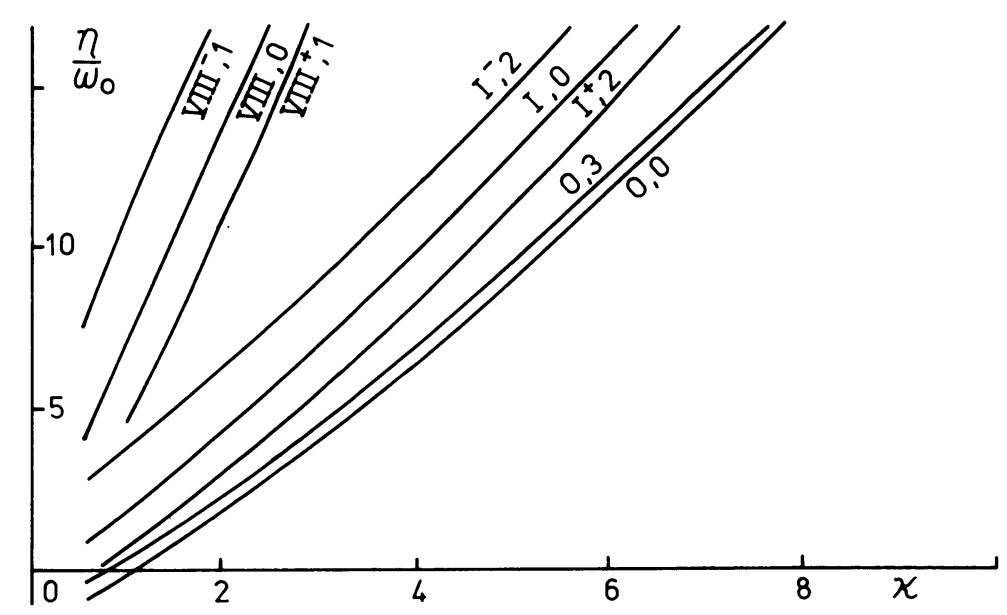

Fig. 2. Reduced damping in function of the reduced wave vector $\kappa$. Conventions are as for Figure 1 .

distributions do give more favorable results. By way of example, and also to indicate the sensitivity of the calculations to the choice of the velocity distribution, we have on Figure 3 also given some curves for the Lorentzian

$$
f^{(0)}(\mathbf{r}, \mathbf{v})=\varrho_{0}(\mathbf{r}) \pi^{-2} \sigma(\mathbf{r})\left(v^{2}+\sigma^{2}\right)^{-2} .
$$

The physical mechanism underlying the preferential propagation inwards appears directly when one analyses how the Landau damping of density waves is affected by the presence of the radial mean field. Landau damping results from the interaction of the density wave and the group of resonant stars, travelling at approximately the phase 
velocity of the wave: the faster particles give up energy to the wave, while the more numerous slower stars absorb energy, the net result being a damping of the wave. If in addition there is a field, the wave will also interact with the field, through the group of resonant stars. For simplicity, consider a radial wave. If the wave is directed inwards, the stars with which it is interacting are being accelerated by the field: the wave thus on average picks up energy from the field and its damping is reduced. If the wave is

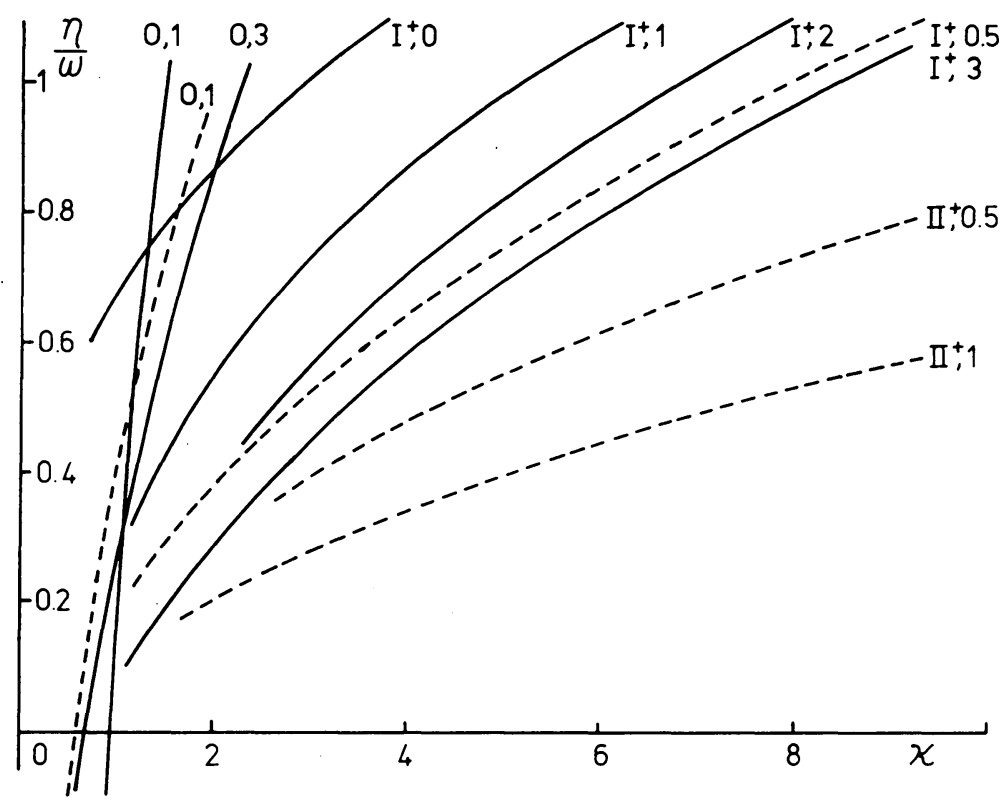

Fig. 3. Relative damping. The dashed lines give the curves for a lorentzian distribution. Other conventions are as for Figure 1. No curves are given for the '-' modes, for which $\eta / \omega>1$.

travelling outwards, the converse occurs: the field exerces a decelerating force on the resonant stars, and the damping of the wave is increased.

The field has also, and predictably, a favorable influence upon the stability of the system. The critical value of the wave vector $\kappa_{c}$ is that for which the damping changes sign. It can be seen, from Figure 2 or Figure 3, that the ' 0 ' mode is here determinant, and that $\kappa_{c}$ is reduced as $\varepsilon$ increases. It should finally be remarked that $\kappa_{c}$, like all the parameters in this problem, is position dependent. (In fact, it is only for small $r$ that one can extend the validity of the calculation down to $\kappa \simeq \kappa_{c}$ without violating the scaling condition (3)). The local character of the stability criterion makes it possible for an inwardly propagating perturbation to excite an instability only in the inner region of the system. Thus while in a purely local description the field enhances stability it may have a destabilizing influence on a larger scale. Insofar as our linear analysis remains valid, such an instability would remain restricted to the central part of the system, since all outwardly propagating vibrations are very strongly damped. 


\section{References}

Lynden-Bell, D. : 1962, Monthly Notices Roy. Astron. Soc. 124, 279.

Severne, G. and Kuszell, A.: 1975, Astrophys. Space Sci. (to appear, denoted as Ref. I).

\section{DISCUSSION}

Feix: The question can be raised about the possibility of treating an homogeneous self gravitating system with a steady state field since this self consistent steady field will be due to the inhomogeneity and the two terms are two aspects of the same phenomenon (finiteness of self gravitating systems). Taking only very small wavelength can be the answer but in that case the field effect is unimportant. Extending the results to the interesting wavelengths (of the system order of length) is of course forbidden.

Severne: Force and inhomogeneity effects can be separated because we have a third parameter at our disposal: the wavelength $\lambda$ of the perturbation. For $\lambda \ll L_{H}$ the separation can be made. The problem is the meaning of the symbol ' $\ll$ '. For Plummer's model, $\gamma \ll L_{H}$ implies $\kappa \gg 0.37\left(1+\left(4 r^{2} / r_{\max }^{2}\right)\right)$, with $r_{\max }$ taken as the effective radius of the system and including 0.72 of the total mass. 